\title{
Will Remdesivir Reshape Cardiovascular Practice in COVID 19 Era?
}

\author{
A. Mohammed Idhrees, MCh, FAIS ${ }^{10}$ Prasanna Karthik Suthakaran, MD, MRCP2 \\ George Jose Valooran, MCh $^{3}$ Mohamad Bashir, MD, PhD, MRCS ${ }^{4}$
}

\author{
${ }^{1}$ Institute of Cardiac and Aortic Disorders, SRM Institutes for Medical \\ Science (SIMS Hospital), Chennai, Tamil Nadu, India \\ 2 Department of General Medicine, Saveetha Medical College \\ Hospital, Chennai, Tamil Nadu, India \\ ${ }^{3}$ Department of CVTS, Rajagiri Hospital, Kochi, Kerala, India \\ ${ }^{4}$ Department of Vascular and Endovascular Surgery, Royal Blackburn \\ Teaching Hospital, Blackburn, Lancashire, United Kingdom of Great \\ Britain and Northern Ireland
}

Int J Angiol 2021;30:155-159.
Address for correspondence A. Mohammed Idhrees, MBBS, MS, MCh, FAIS, Institute for Cardiac and Aortic Disorders, SRM Institutes for Medical Science (SIMS Hospitals), Chennai, Tamil Nadu 600026, India (e-mail: a.m.idhrees@gmail.com).

\begin{abstract}
Keywords

- remdesivir

- COVID-19

- coronavirus

- SARS-CoV2

- cardiovascular surgery

- cardiovascular medicine

Infection with the novel coronavirus, SARS-CoV2, produces the clinical syndrome COVID-19. COVID-19 is a systemic illness inducing hyperinflammation and cytokine storm affecting multiple organs including the myocardium which is reflected in elevated cardiac biomarkers such as troponin, lactate dehydrogenase, and creatinine kinase MB. Furthermore, COVID-19 has been implicated in increased predilection to thromboembolic phenomena. Hence, mortality in patients with associated cardiovascular disease has been higher compared with the cohort with no cardiovascular comorbidity. It is entirely unknown how remdesivir will change the facet of cardiovascular medicine and surgery. In the present constantly changing climate, this review of remdesivir and its association with cardiovascular disease is comprehensive as of June 17, 2020 and it highlights the science behind this drug and its potential implications to cardiovascular practice.
\end{abstract}

COVID-19, caused by the SARS-CoV2, originated in December 2019 and was declared a pandemic in March 2020. The disease has claimed close to half a million lives in the span of 6 months. Several therapeutic agents have been appraised for the treatment of COVID-19, but none has shown to be effective. Remdesivir, an inhibitor of the viral RdRp, has shown some early promising results in the treatment of COVID-19. ${ }^{1}$ Data on the effect and the ill effects of the drug in cardiac patients are lacking. In these unprecedented times, cardiac surgical patients are exposed to the potential risks of COVID-19, such as precarious effect of cardiopulmonary bypass, stress response to surgery, and the associated systemic inflammatory response and potential end-organ damage. Hence, the trend of practical decision is to divert patients to cardiological interventions to alleviate coronary disease. Patients with coronary artery disease appear to share the same comorbidities as those with COVID-19. A large Chinese study analyzing data of 44,672 confirmed COVID-19 cases revealed $12.8 \%$ had hypertension, 5.3\% diabetes mellitus, and $4.2 \%$ cardiovascular disease (CVD). ${ }^{2} \mathrm{~A}$ further study of 5,700 patients from the U.S. reported a similar message that hypertension (56.6\%), obesity (41.7\%), diabetes $(33.8 \%)$, coronary artery disease $(11.1 \%)$, and congestive heart failure (6.9\%) were common comorbidities in patients with COVID-19. ${ }^{3}$ It is becoming clear that cardiac involvement is both prevalent and prognostic in COVID-19. The most important question asked, would remdesivir and its promising potentials return the mainstream for cardiac surgery interventions and when would this be applicable? Here in our review we assess the effect of such drugs on the published online

December 3, 2020 (c) 2020. International College of Angiology. All rights reserved. Thieme Medical Publishers, Inc., 333 Seventh Avenue, 18th Floor, New York, NY 10001, USA
DOI https://doi.org/ 10.1055/s-0040-1721403. ISSN 1061-1711. 
current understanding of remdesivir and its implications on cardiac disease.

\section{The Inherent of Risk}

In an attempt to prevent cardiac surgery patients from encountering COVID-19, we might have to defer elective or semielective operations into emergent ones that may pose an even greater risk on patients' outcomes. It is becoming imperative that prudent decisions or rather, a balanced decision framework between performing cardiac surgery potentiating the risk for COVID-19 encounter versus the undesired course of delaying surgery or deferring our patient population for percutaneous interventions is the need of the hour. Compounding this decision framework is the lack of robust evidence to support either notion. Moreover, we ought to shed light on the potential possibility of asymptomatic patients with COVID-19 infection who are a particular risk group and entity. Asymptomatic infection at the time of laboratory confirmation of COVID-19 has been widely reported, with a large proportion of these cases experiencing some symptoms at a later stage of infection. No vaccine or specific antiviral treatment for COVID-19 has yet been shown to be effective; hence, supportive therapeutics to ameliorate and protect multiorgan function may be beneficial. The constantly changing clinical scenario of COVID-19 patients is modifying our understanding of this disease on a daily basis.

\section{Cardiac Biomarkers in COVID-19}

CVD is the third most prevalent comorbidity in SARS-CoV2 patients, and the severity of infection correlated with a higher risk of CVD (odds ratio 3.42, 95\% confidence interval [CI] 1.88-6.22). ${ }^{4}$ From the early reports, $25 \%$ of COVID-19related intensive care unit (ICU) admissions had background CVD. If hypertension is also accounted for, this figure rises to a staggering $58 \%,{ }^{5}$ which puts more than half of COVID-19related ICU admissions at risk for cardiovascular complications. SARS-CoV2 infection superimposed on existing CVD results in rapid deterioration of clinical course. Angiotensinconverting enzyme (ACE) II, which is the route of entry for SARS-CoV2 into the cell, is significantly expressed in myocardium, thus putting the cardiovascular system at risk for direct damage by the virus, like the lung alveolar cells. ${ }^{6}$

Myocardial injury has a significant association in SARSCoV2 infection and it occurs by two mechanisms, namely (1) systemic inflammatory response consequential to the cytokine storm with associated elevations of interleukin (IL)-6, Ddimer, lactate dehydrogenase (LDH), and ferritin and (2) direct virus-mediated myocardial injury mediated by ACE II. $^{7}$ The associated heart failure is a surrogate marker for mortality with its incidence of $51.9 \%$ in fatal cases whereas only $11.7 \%$ of survivors of COVID-19 had features of heart failure. This forms the basis of using cardiac biomarkers like high-sensitivity cardiac troponin I (cTnI), brain natriuretic peptide (BNP), and creatinine kinase-MB (CK-MB) for assessing the severity and prognostication of SARS-CoV2 infection. ${ }^{8}$
Several reports have evaluated the predictive value of cTnI in foretelling the clinical deterioration associated with SARSCoV2 infection..$^{8-11}$ These reports stated that the high values of cTnI could potentially predict case severity, and in a metaanalysis, the mean difference in the values of cTnI between the severe and nonsevere cases was found to be $25.6 \mathrm{ng} / \mathrm{mL}$ (95\% CI $6.8-44.5 \mathrm{ng} / \mathrm{mL}){ }^{6}$ The case fatality rate was also higher in patients with higher cTnI values (51.2\% vs. $4.5 \%$ ), which signified the potential fatality of myocardial injury in the setting of COVID-19 infection. ${ }^{9}$ With regards to cardiac troponin T (cTnT), another marker for myocardial injury, Guo et al reported its prognostic value with a mortality rate of $59.6 \%$ in patients with cTnT elevation versus $8.9 \%$ in patients with normal values. ${ }^{11}$ Several investigators have reported the concomitant rise of BNP paralleling cTnT and cTnI levels pointing to its relevance in prognostication. ${ }^{10,11}$ This marker of cardiac failure usually rises as the pump failure secondary to myocardial injury sets in and its elevation has been correlated with a case fatality of up to $51.2 \%{ }^{11}$ Thus, serial BNP levels help to assess the course of clinical deterioration.

CK-MB levels again a marker of myocardial injury has been reported to be positively correlating with $\mathrm{cTnI}$ levels and has a significant association within hospital deaths $(p<0.001) .^{8}$ However, there is conflicting evidence with regards to CK$\mathrm{MB}$ as some studies have shown no association between CKMB levels and critically ill SARS-CoV2 patients. ${ }^{12}$ Similarly, evidence supporting BNP as a predictor of adverse events in COVID-19 infections is not conclusive. ${ }^{13}$ The only consistent cardiac biomarker which can be of prognostic use would be cTnI in the background of SARS-CoV2 infection as evidenced from recent literature. However, $\mathrm{cTnI}$ is found to be elevated in sepsis/systemic inflammatory response syndrome, pneumonia, acute respiratory distress syndrome, and chronic obstructive pulmonary disease which are documented clinical presentations of SARS-CoV2. Thus, the evidence makes cTnI a useful prognostic marker for COVID-19 but not specific to myocardial injury. ${ }^{13}$

\section{The Thrombotic Problem in SARS-CoV2}

The SARS-CoV2 infection has a disproportionately high incidence of venous thromboembolism as well as arterial thrombosis. More than one-third of infected cases (35-45\%) have some form of thromboembolic complication. ${ }^{14}$ The hematological picture seen in SARS-CoV2 infection with the thrombotic problem is that of a low-grade disseminated intravascular coagulation (DIC) with thrombotic microangiopathy. ${ }^{15}$ D-dimer values in SARS-CoV2 infection with thrombus load is considerably elevated (several times above the cut-off value of $0.5 \mathrm{mg} / \mathrm{dL}$ ). The prognostic value of high D-dimer concentrations is established by reports stating the mortality and requirement of ICU admissions in patients with higher D-dimer concentrations is higher when compared with patients with normal values. ${ }^{16}$ However, the Ddimer elevation in SARS-CoV2 is exceptional in the fact that the elevations are way higher than what is seen in DIC associated with sepsis. ${ }^{15}$ The thrombocytopenia associated with SARS-CoV2 is categorized as mild $(<150,000 / \mathrm{dL}$, with 
only $5 \%$ with counts $<100,000 / \mathrm{dL}$ ) whereas that associated with DIC usually severe. ${ }^{17}$ Similarly, the mean prothrombin time also showed only a mild elevation by few seconds (15.6 vs. 13.6 seconds) between survivors and nonsurvivors with SARS-CoV2 infection which is again in contrast to what is seen with DIC. ${ }^{18}$ All these factors point to a clinical picture that is distinct from classical DIC with SARS-CoV2 infection.

Serum ferritin levels are found to be quite high in SARS-COV2 infection with thrombotic complications. However, the markers of hemolysis like serum LDH levels of schistocytes in the peripheral film are absent from this problem. ${ }^{16}$ These features point to a primarily thrombotic microangiopathy in SARS-CoV2 infection which is most marked in pulmonary capillaries adding to the clinical deterioration of the patients. ${ }^{15,18}$

The cytokine storm associated with the infection comprising of tumor necrosis factor- $\alpha$ and IL- 1 and IL- 6 cause endothelial inflammation and damage resulting in exposure to tissue factor and activating the coagulation system. ${ }^{15}$ The fibrinolytic system is also activated by endothelial injury secondary to these mediators resulting in higher concentrations of D-dimer and fibrin degradation products. Serial assessment of D-dimer, prothrombin time, and platelet count every second or third day has been recommended for monitoring. ${ }^{15}$ Subcutaneous low molecular weight heparin is effective in treating the prothrombotic state in SARSCoV2 infection and therapeutic anticoagulation is advised if no hematology testing is available. ${ }^{15,18}$

\section{Pharmacology of Remdesivir}

Remdesivir (GS-5734; Gilead Sciences, Inc) is a prodrug that is converted in vitro into the active form called GS-441524 which acts as an adenosine triphosphate analog. The molecule acts as a substrate for viral RdRp and leads to delayed chain termination. The molecule has a 1'-cyano-adenosine analog structure that causes steric hindrance to the RdRp. The molecule also demonstrated a unique advantage in that it was highly selective in its incorporation into the replicating structure over the regular adenosine moiety. ${ }^{19}$ According to Eastman et al, human ribonucleic acid polymerase II (RNAP II) and human mitochondrial RNAP were not inhibited by remdesivir and thereby increasing its selectivity 500 -fold. ${ }^{20}$ Though initially developed as a treatment for hepatitis $C$ virus infection, it has been an effective therapeutic agent against the Nipah virus, Ebola virus, and Marburg virus and other viruses. ${ }^{21}$ Its inhibitory effect against SARS-CoV in 2003 and the MERS-CoV in 2012 has been effective and subsequently was proposed for the treatment of COVID-19 infection. ${ }^{22,23}$

\section{Role of Remdesivir in COVID-19}

The earliest evidence of remdesivir in COVID-19 patients was documented in April 2020. Remdesivir was used on a compassionate basis for 53 patients with severe COVID-19 across nine countries. With a median follow-up of 18 days, there was an improvement in oxygen support class in more than two-thirds (68\%) of the cohort including $57 \%$ of the patients who were weaned off ventilator. ${ }^{24}$ The first trial from China on the use of remdesivir in COVID-19 concluded that the drug was not associated with statistically significant clinical benefits though the patients receiving remdesivir had a faster time to clinical improvement than the placebo arm. The study was underpowered due to the reduction in the recruited population and hence the benefits could not be adequately assessed. ${ }^{1}$ Several trials are being conducted on the efficacy of the drug at present. A search of the National Clinical Trials Registry on June 6, 2020 (www.clinicaltrials. gov), revealed a total of 15 studies involving remdesivir in the management of COVID-19 diseases. Of this, two were not yet recruiting, seven were recruiting, two were active but not recruiting, one was suspended, one was terminated, and one was completed while expanded access was available for two trials. All the active trials had a regime of using intravenous remdesivir at a dose of $200 \mathrm{mg}$ on day 1 followed by $100 \mathrm{mg}$ on days 2 to 10 . The preliminary data from Adaptive Covid-19 Treatment Trial (ACTT-1) were promising. The data showed that among 1,059 patients treated with intravenous remdesivir or placebo, the median recovery time was 4 days shorter in the remdesivir arm (11 vs. 15 days, rate ratio for recovery $1.32 ; 95 \% \mathrm{CI}, 1.12-1.55 ; p<0.001$ ) and had a $31 \%$ faster time to recovery. However, the preliminary data did not support evidence for a reduction in mortality. ${ }^{25}$

Based on the findings of the above trial, the U.S. Food and Drug Administration issued an emergency use authorization for remdesivir for the treatment of COVID-19 in patients with severe disease. ${ }^{26}$ European Medicines Agency has recommended the compassionate use of remdesivir in COVID-19 patients and the statutory approval is pending submission of documents by the parent company, Gilead Lifesciences. ${ }^{27}$ On June 3, 2020, the Drug Controller General of India provided emergency approval for its usage in Indian patients.

\section{Cardiac and Vascular Complications of Remdesivir}

In the early reports of remdesivir usage in COVID19 patients, the incidence of adverse events was more among the patients with invasive ventilation which points to the activation of inflammatory cascade predisposing to thrombosis as explained earlier. Hypotension is one of the more common events with the incidence of atrial fibrillation being $6 \%$ and deep vein thrombosis (DVT) being $9 \%{ }^{24}$

In the randomized trial by Wang et al, remdesivir usage was associated with hypokalemia and hyponatremia. The incidence of cardiopulmonary failure was similar in both arms. There was an increased number of patients in the remdesivir group who discontinued the drug due to adverse events. Drug withdrawal was in three patients in the intervention arm and one patient in the control arm. Incidences of DVT and pulmonary thromboembolism were similar in both the arms. ${ }^{1}$ In the preliminary data from ACTT-1 trial, serious atrial fibrillation was reported in $0.7 \%(n=4)$ patients in the remdesivir arm compared with $0.4 \%(n=2)$ patients in the placebo arm, while nonserious atrial fibrillation was reported in $0.6 \%(n=3)$ patients in the remdesivir arm compared with $1.1 \%(n=6)$ patients in the placebo arm. ${ }^{25}$ 


\section{Cardiac Drug Interactions}

The current knowledge about the metabolism of remdesivir indicates that it undergoes extensive first-pass metabolism by the liver and hence an oral formulation is quite unlikely to be effective. ${ }^{28}$ The drug is predominantly metabolized by hydrolases and excreted in the urine. Remdesivir is a substrate of multiple cytochrome P450 enzymes including CYP2C8, CYP2D6, and CYP3A4, but clinically significant interactions between remdesivir and CYP3A4 inhibitors or inducers are highly unlikely. ${ }^{29}$ Caution is to be advised when coadministering amiodarone, especially in patients with a creatinine clearance of less than $30 \mathrm{~mL} / \mathrm{min} .{ }^{30}$ Since it is a drug still undergoing trials, not much about potential drug interactions are known. In clinical trials so far no potentially, deleterious effects have been noted due to interactions with cardiac drugs, but more studies are needed before concluding definite cardiac safety.

\section{A Call Out for Revamping Evidence}

One of the major concerns to be addressed with remdesivir use is the possible cumulative potential for thromboembolic complications superimposed on the thrombotic problem of SARS-CoV2 infection. Although there is limited evidence that remdesivir is associated with vascular thrombosis, the coupled effects of arterial and venous thrombosis would raise concerns in the following settings:

(1) Potential for stent thrombosis secondary to increased platelet aggregation in patients undergoing percutaneous coronary intervention with acute coronary syndrome necessitating thrombolytic therapy. ${ }^{31}$

(2) Early graft failures predisposing to perioperative myocardial infarction in patients undergoing coronary artery bypass grafting.

(3) Increased risk of DVT and pulmonary embolism, in SARS-CoV2 patients undergoing cardiac surgery on remdesivir therapy.

(4) Cautious use of vascular endografts in aortic and peripheral vascular surgeries with the potential for early graft thrombosis.

The evidence for ideal antiplatelet therapy is still lacking in these patients. The authors suggest an aggressive dual antiplatelet therapy and a strict vigilance on the electrolytes in these patients till the evidence emerge.

Hypokalemia associated with remdesivir may precipitate cardiac arrhythmias and the potential for interaction with amiodarone, one of the more commonly used drugs in clinical practice, certainly ring bells to any cardiac surgeon. With the extension of drug usage, more evidence may emerge in the future.

\section{Conclusion}

"COVID-19 pandemic" is an unprecedented challenge to the medical fraternity of today. The current ideas and notions of clinical decision making have been found inadequate in view of the pandemic. Guidelines are being updated every day and newer evidence is being generated on a daily basis. In this scenario, remdesivir has shown some potential as a therapeutic option. More evidence is needed before accepting it as a standard of care keeping in mind that it is still in the evaluation stage as any other medicine and it has some more hurdles to clear before it should make a definite impact on our clinical practice.

\section{Conflict of Interest}

None declared.

\section{References}

1 Wang Y, Zhang D, Du G, et al. Remdesivir in adults with severe COVID-19: a randomised, double-blind, placebo-controlled, multicentre trial. [published correction appears in Lancet. 2020 May 30;395(10238):1694]. Lancet 2020;395(10236):1569-1578

$2 \mathrm{Wu}$ Z, McGoogan JM. Characteristics of and important lessons from the coronavirus disease 2019 (COVID-19) outbreak in China: summary of a report of 72314 cases from the Chinese Center for Disease Control and Prevention. JAMA 2020;323(13):1239-1242

3 Richardson S, Hirsch JS, Narasimhan M, et al; the Northwell COVID19 Research Consortium. Presenting characteristics, comorbidities, and outcomes among 5700 patients hospitalized with COVID-19 in the New York City area. JAMA 2020;323(20):2052-2059

4 Yang J, Zheng Y, Gou X, et al. Prevalence of comorbidities in the novel Wuhan coronavirus (COVID-19) infection: a systematic review and meta-analysis. Int J Infect Dis 2020;94:91-95

5 Wang D, Hu B, Hu C, et al. Clinical characteristics of 138 hospitalized patients with 2019 novel coronavirus-infected pneumonia in Wuhan, China. JAMA 2020;323(11):1061-1069

6 Guo J, Huang Z, Lin L, Lv J. Coronavirus disease 2019 (COVID-19) and cardiovascular disease: a viewpoint on the potential influence of angiotensin-converting enzyme inhibitors/angiotensin receptor blockers on onset and severity of severe acute respiratory syndrome coronavirus 2 infection. J Am Heart Assoc 2020;9 (07):e016219

7 Clerkin KJ, Fried JA, Raikhelkar J, et al. Covid-19 and cardiovascular disease. Circulation 2020;141(20):1648-1655

8 Zhou F, Yu T, Du R, et al. Clinical course and risk factors for mortality of adult inpatients with COVID-19 in Wuhan, China: a retrospective cohort study. Lancet 2020;395(10229):1054-1062

9 Xu Z, Shi L, Wang Y, et al. Pathological findings of COVID-19 associated with acute respiratory distress syndrome. Lancet Respir Med 2020;8(04):420-422

10 Shi S, Qin M, Shen B, et al. Association of cardiac injury with mortality in hospitalized patients with COVID-19 in Wuhan, China. JAMA Cardiol 2020;5(07):802-810

11 Guo T, Fan Y, Chen M, et al. Cardiovascular implications of fatal outcomes of patients with coronavirus disease 2019 (COVID-19). JAMA Cardiol 2020;5(07):811-818

12 Wan S, Xiang Y, Fang W, et al. Clinical features and treatment of COVID-19 patients in northeast Chongqing. J Med Virol 2020;92 (07):797-806

13 Aboughdir M, Kirwin T, Abdul Khader A, Wang B. Prognostic value of cardiovascular biomarkers in COvid-19: a review. Viruses 2020; 12(05):527

14 Klok FA, Kruip MJHA, van der Meer NJM, et al. Incidence of thrombotic complications in critically ill ICU patients with COVID-19. Thromb Res 2020;191:145-147

15 Levi M, Thachil J, Iba T, Levy JH. Coagulation abnormalities and thrombosis in patients with COVID-19. Lancet Haematol 2020;7 (06):e438-e440

16 Tang N, Li D, Wang X, Sun Z. Abnormal coagulation parameters are associated with poor prognosis in patients with novel coronavirus pneumonia. J Thromb Haemost 2020;18(04):844-847 
17 Huang C, Wang Y, Li X, et al. Clinical features of patients infected with 2019 novel coronavirus in Wuhan, China. Lancet 2020;395 (10223):497-506

18 Tang N, Bai H, Chen X, Gong J, Li D, Sun Z, et al. Anticoagulant treatment is associated with decreased mortality in severe coronavirus disease 2019 patients with coagulopathy. J Thromb Haemost 2020;18(05):1094-1099

19 Gordon CJ, Tchesnokov EP, Woolner E, et al. Remdesivir is a directacting antiviral that inhibits RNA-dependent RNA polymerase from severe acute respiratory syndrome coronavirus 2 with high potency. J Biol Chem 2020;295(20):6785-6797

20 Eastman RT, Roth JS, Brimacombe KR, et al. Remdesivir: a review of its discovery and development leading to emergency use authorization for treatment of COVID-19. ACS Cent Sci 2020;6 (05):672-683

21 Lo MK, Jordan R, Arvey A, et al. GS-5734 and its parent nucleoside analog inhibit Filo-, Pneumo-, and Paramyxoviruses. Sci Rep 2017;7:43395

22 de Wit E, Feldmann F, Cronin J, et al. Prophylactic and therapeutic remdesivir (GS-5734) treatment in the rhesus macaque model of MERS-CoV infection. Proc Natl Acad Sci US A 2020;117(12):6771-6776

23 Wang M, Cao R, Zhang L, et al. Remdesivir and chloroquine effectively inhibit the recently emerged novel coronavirus (2019-nCoV) in vitro. Cell Res 2020;30(03):269-271
24 Grein J, Ohmagari N, Shin D, et al. Compassionate use of remdesivir for patients with severe Covid-19. N Engl J Med 2020;382 (24):2327-2336

25 Beigel JH, Tomashek KM, Dodd LE, et al; ACTT-1 Study Group Members. Remdesivir for the treatment of Covid-19-preliminary report. N Engl J Med 2020. Doi: 10.1056/NEJMoa2007764

26 Accessed June 6, 2020 at: https://www.fda.gov/news-events /press-announcements/coronavirus-covid-19-update-fda-issues -emergency-use-authorization-potential-covid-19-treatment

27 Accessed June 6, 2020 at: https://www.ema.europa.eu/en/human-regulatory/overview/public-health-threats/coronavirusdisease-covid-19/treatments-vaccines-covid-19

28 Siegel D, Hui HC, Doerffler E, et al. Discovery and synthesis of a phosphoramidate prodrug of a pyrrolo[2,1-f][triazin-4-amino] adenine C-nucleoside (GS-5734) for the treatment of Ebola and emerging viruses. J Med Chem 2017;60(05):1648-1661

29 McCreary EK, Pogue JM. Coronavirus disease 2019 treatment: a review of early and emerging options. Open Forum Infect Dis 2020;7(04):a105

30 Yang K. What do we know about remdesivir drug interactions? Clin Transl Sci 2020;13(05):842-844

31 Akhmerov A, Marbán E. COVID-19 and the heart. Circ Res 2020; 126(10):1443-1455 